Pacific Journal of Mathematics

QUASI PROJECTILES IN ABELIAN AND MODULE 


\title{
QUASI PROJECTIVES IN ABELIAN AND MODULE CATEGORIES
}

\author{
K. M. RANGASWAMY AND N. VANAJA
}

If $R$ is a ring without zero divisors then it is shown that any torsion-free quasi-projective left $R$-module $A$ is projective provided $A$ is finitely generated or $A$ is "big". It is proved that the universal existence of quasi-projective covers in an abelian category with enough projectives always implies that of the projective covers. Quasi-projective modules over Dedekind domains are described and as a biproduct we obtain an infinite family of quasi-projective modules $Q$ such that no direct sum of infinite number of carbon copies of $Q$ is quasi projective. Perfect rings are characterised by means of quasi-projectives. Finally the notion of weak quasi-projectives is introduced and weak quasi-projective modules over a Dedekind domain are investigated.

1. Introduction. An object $A$ in a category $\mathscr{A}$ is called quasiprojective [14] if given an epimorphism $A \stackrel{f}{\rightarrow} B$ and a morphism $g: A \rightarrow B$, there is $h: A \rightarrow A$ making the following diagram

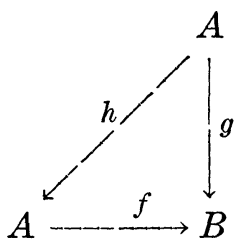

commutative. This paper starts with the investigation of the quasiprojectives in an abelian category. Utilising a few basic lemmas, it is shown that the universal existence of the quasi-projective covers in an abelian category $\mathscr{A}$ implies that of the projective covers, provided $\mathscr{A}$ possesses enough projectives and this answers affirmatively a question of Faith [4] in a general form. Next we consider quasi-projectives in the category of modules. It turns out that "big" torsion-free quasi-projectives over rings without zero divisors are always projective. Artin semi-simple rings are characterised as those rings over which quasi-projectives and projectives coincide. In $\S 5$, quasi-projectives over a Dedekind domain $R$ are investigated: A quasiprojective $R$-module is either torsion or torsion-free. A torsion $R$ module is quasi-projective if and only if it is quasi-injective but not 
injective. If $R$ is a complete discrete valuation ring, then the torsionfree quasi-projective $R$-modules are just the free $R$-modules and the torsion-free modules of finite rank. Suppose $R$ is a Dedekind domain which is not a complete discrete valuation ring and $\sigma$ is the number of distinct prime ideals of $R$. If $\sigma \leqq 2^{\aleph_{0}}$, then all the torsion-free $R$-quasi-projectives are projective. If $\sigma>2^{\aleph_{0}}$, then a torsion-free quasi-projective $R$-module $A$ is projective if either (i) rank $A \leqq \boldsymbol{\aleph}_{0}$ or (ii) $\operatorname{rank} A>\sigma$. In the case when $\boldsymbol{\aleph}_{0}<\operatorname{rank} A<\sigma, A$ is torsionless, $\aleph_{1}$-projective and contains a free summand $F$ having the same rank as $A$. As a biproduct we at once get an infinite family of quasi-projective modules $A$ such that no direct sum of infinite number of copies of $A$ is quasi-projective. In $\S 6$, Perfect rings are characterised as those rings $R$ such that $R$-quasi-projectivity survives under direct limits. $A$ weakened form of quasi-projectivity - called weak quasi-projectivity - is considered in the last section and weak quasi-projectives over a Dedekind domain are completely characterised.

2. Preliminaries. All the rings that we consider are associative and are assumed to possess an identity and all the modules unitary left modules. A sub-module $S$ of an $R$-module $M$ is called fully invariant if $S$ is stable under every $R$-endomorphism of $M . \quad S$ is called a small submodule, if $S+T=M$ implies $T=M$ for any submodule $T$ of $M$. A projective module $P$ is called a projective cover of $M$ if there is an epimorphism $P \rightarrow M$ whose kernel is small. A module $M$ over an integral domain is called reduced if 0 is the only divisible submodule of $M$. By the rank of a torsion-free module $M$ over a Dedekind domain $R$ we shall mean the cardinality of a maximal $R$ independent subset of $M$. An $R$-module $M$ is called quasi-injective if for any exact sequence $0 \rightarrow S \stackrel{i}{\rightarrow} M$, the induced sequence

$$
\operatorname{Hom}_{R}(M, M) \stackrel{i *}{\longrightarrow} \operatorname{Hom}_{R}(S, M) \longrightarrow 0
$$

is exact, where $i^{*}(f)=i \circ f$ for all $f$ in $\operatorname{Hom}_{R}(M, M)$. For the basic results in category theory, modules and abelian groups, the reader is referred to [5], [6], [10] and [11].

3. Quasi-projectivity in abelian categories. In this section, we examine the properties of quasi-projective objects in an abelian category. The main result shows that the universal existence of quasi-projective covers in an abelian category $\mathscr{A}$ implies that of projective covers, provided $\mathscr{A}$ possesses enough projectives.

Note. In conformity with our notation in the subsequent sections, a composite $f \circ g$ of two morphisms is obtained by applying $f$ 
first and then $g$.

LEMMA 3.1 [14]. In an abelian category, any retract of a quasiprojective is quasi-projective.

The following lemma gives a condition under which an object becomes projective.

Lemma 3.2. An object $A$ in an abelian category is projective if and only if there exists an epimorphism $P \rightarrow A$ with $P$ projective and $A \oplus P$ is quasi-projective.

Proof. We prove only the "if" part. Let $f: P \rightarrow A$ be the given epimorphism, $\quad A \stackrel{i}{\rightarrow} A \oplus P \stackrel{j}{\rightarrow} A=1_{A} \quad$ and $\quad P \stackrel{i^{\prime}}{\rightarrow} A \oplus P \stackrel{j^{\prime}}{\rightarrow} P=1_{P} . \quad$ By the quasi-projectivity of $A \oplus P$, there exists $g: A \oplus P \rightarrow A \oplus P$ such that $A \oplus P \stackrel{j}{\rightarrow} A=A \oplus P \stackrel{g}{\rightarrow} A \oplus P \stackrel{j^{\prime}}{\rightarrow} P \stackrel{f}{\rightarrow} A$. Then

$$
1_{A}=i j=i\left(g \circ j^{\prime} \circ f\right)=\left(i \circ g \circ j^{\prime}\right) f \text {. }
$$

Thus $A$ is a retract of $P$ and hence projective.

Dualizing 3.2, we obtain

Lemma 3.2'. An object $A$ is an abelian category is injective if and only if there is a monomorphism $A \rightarrow I$ with $I$ injective and $A \oplus I$ is quasi-injective.

Next we examine the universal existence of quasi-projective covers.

DeFinition 3.3. ( $\mathrm{i}$ ) An epimorphism $f$ in a category is called a minimal epimorphism if, whenever $g \circ f$ is an epimorphism, $g$ itself is an epimorphism.

(ii) $A \rightarrow X$ is called a projective (quasi-projective) cover in a category, if $A$ is projective (quasi-projective) and $f$ is a minimal epimorphism.

(iii) A category $\mathscr{A}$ is called perfect (quasi-perfect) if every object in $\mathscr{A}$ possesses a projective (quasi-projective) cover.

(iv) A category is said to possess enough projectives, if, to every object $A$, there is an epimorphism $P \rightarrow A$ with $P$ projective.

REMARK. (i) For an axiomatic treatment of minimal epimorphisms see [1]. Observe that in the category of $R$-modules, an epimorphism $f: A \rightarrow B$ is minimal if and only if $\operatorname{Ker} f$ is small in A. 

[3].

(ii) The notion of a perfect category has been considered in [2],

(iii) Our definition of a quasi-projective cover is slightly different from the one defined in [14] for modules. However, it is easy to see that for the category of modules over a ring $R$, the universal existence of quasi-projective covers according to the new definition is equivalent to the universal existence of quasi-projective covers according to the definition given in [14].

It is clear that a perfect abelian category is quasi-perfect. Conversely, is a quasi-perfect abelian category perfect? This is the category-theoretical formulation of a question raised by C. Faith $[4]^{11}$. The following theorem answers this:

THEOREM 3.4. An abelian category $\mathscr{A}$ is perfect if and only if it is quasi-perfect and possesses enough projectives.

Proof. IF part: Let $A \in \mathscr{A}$ and $P \stackrel{u}{\rightarrow} A$ an epimorphism with $P$ projective. Let $g: Q^{\prime} \rightarrow A \oplus P$ be a quasi-projective cover of $A \oplus P$. Consider the following commutative diagram

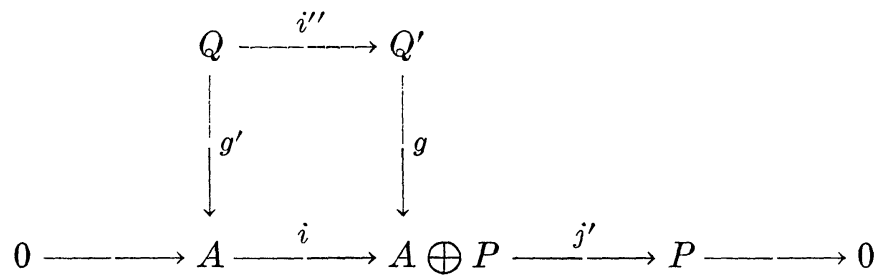

where the square is a pull-back and

$$
A \stackrel{i}{\longrightarrow} A \oplus P \stackrel{j}{\longrightarrow} A=1_{A}, P \stackrel{i^{\prime}}{\longrightarrow} A \oplus P \stackrel{j^{\prime}}{\longrightarrow} P=1_{P} \text {. }
$$

By Lemma 2.61 of [5],

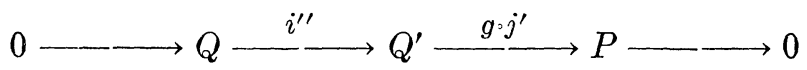

is an exact sequence which splits since $P$ is projective. Let $f: P \rightarrow Q^{\prime}$ be such that $f \circ g \circ j^{\prime}=1_{P}$. Since $g$ is epic and the square is a pullback, $g^{\prime}$ is also epic. We claim $g^{\prime}$ is minimal. Let $h^{\prime}: C \rightarrow Q$ be such that $h^{\prime} \circ g^{\prime}$ is epic. Let $h=\left(h^{\prime} \circ i^{\prime \prime}\right) \oplus f$. Consider the following commutative diagram

1) While this paper was being written we found out that this question has been recently answered independently by A. Koehler [12], K. R. Fuller, D. A. Hill and J. Golan for the category of $R$-modules. 


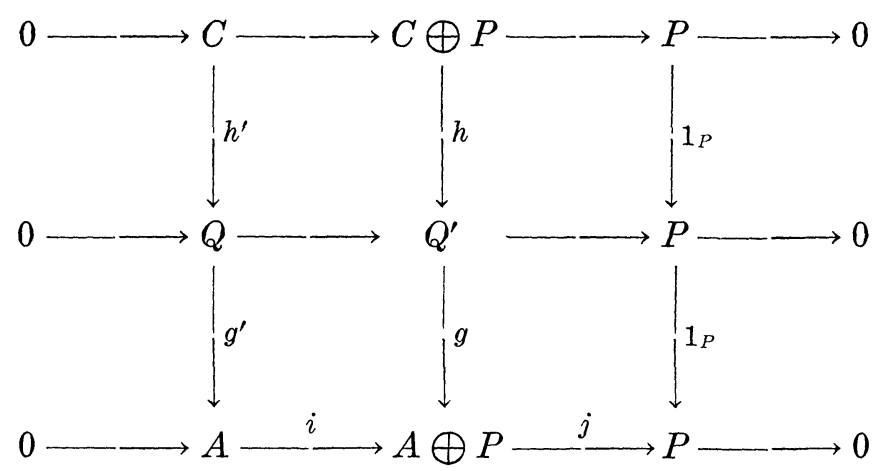

where the top row is split exact with the obvious maps. By the 5lemma, $h \circ g$ is epic and since $g$ is minimal, $h$ is epic. Since

$$
C \longrightarrow C \oplus P \longrightarrow P \longrightarrow 0
$$

is exact, again by Lemma 2.61 of [5], the left top square is a pullback. Since $h$ is epic, $h^{\prime}$ is also epic. Thus $g^{\prime}$ is minimal epic. Since $P$ is projective and $u: P \rightarrow A$, there exists $v: P \rightarrow Q$ such that $v \circ g^{\prime}=u$. By the minimality of $g^{\prime}, v$ is an epimorphism. Then the quasi-projectivity of $Q \oplus P$ and the Lemma 3.2 imply that $Q$ is projective. Thus $g^{\prime}: Q \rightarrow A$ is a projective cover of $A$ and we conclude that the category is perfect.

REMARK 1. Theorem 3.4 is best possible in the sense that it fails to be true if $\mathscr{A}$ is not an abelian category. To see this, let $\mathscr{A}_{b}$ be the category of all the abelian groups and $\mathscr{A}$ the full subcategory of $\mathscr{A}_{b}$ consisting of all the cyclic groups. Then $\mathscr{A}$ is not abelian. $\mathscr{A}$ has enough projectives and is clearly quasi-perfect (every object in $\mathscr{A}$ is quasi-projective). But $\mathscr{A}$ is not perfect since the prime cyclic group $Z(p)$ possesses no projective cover in $\mathscr{A}$.

REMARK 2. A quasi-perfect abelian category need not possess enough projectives. The category $\mathscr{F}_{p}$ of all finite abelian $p$-groups is one such. The quasi-projectives in $\mathscr{F}_{p}$ are the direct sums of isomorphic cyclic $p$-groups [7]. $\mathscr{F}_{p}$ is abelian and is readily seen to be quasi-perfect. But it possesses no non-trivial projectives.

4. Quasi-projectives in the category of modules. In this section we indicate some of the simple properties of quasi-projective modules over a ring. We also investigate when a quasi-projective module over a ring $R$ without zero-divisors becomes projective. It turns out in a surprisingly simple way that the "big" torsion-free quasi-projectives over such $R$ are projective. Some of the preliminary lemmas in this section hold in any abelian category but, for the sake 
of convenience, we will consider only the module case. Lemmas 4.3 and 4.4 occur in [7], but are proved here for the same of completeness.

LEMMA 4.1. [14]. If $A$ is a quasi-projective $R$-module and $S$ is fully invariant in $A$, then $A / S$ is quasi-projective.

Corollary. Let $I$ be a two sided ideal of a ring $R$. Then $R / I$ is quasi-projective as an $R$-module.

The converse of Lemma 4.1 is not always true. It holds, however, under some restriction on $S$, as indicated below.

LEMmA 4.2. Let $S$ be a small submodule of a quasi-projective module $A$. Then $A / S$ is quasi-projective if and only if $S$ is fully invariant in $A$.

To prove this, replace the word, "projective" in the proof of proposition 2.2 of [14] by "quasi-projective".

The following lemma gives a condition when a submodule of a quasi-projective module becomes a summand.

Lemma 4.3. Let $S$ be a submodule of a quasi-projective module $A$. Then $S$ is a summand if and only if $A / S$ is isomorphic to a summand of $A$.

Proof. Let $A=B \oplus C$ and $f: B \rightarrow A / S$ be an isomorphism. Define $g: A \rightarrow A / S$ by $g \mid B=f$ and $g \mid C=0^{2)}$. By the quasi-projectivity of $A, g$ lifts to an endomorphism $h$ of $A$ such that $h \circ p=g$, where $p: A \rightarrow A / S$ is the natural map. Set $p^{\prime}=f^{-1} \circ h$. Since $p^{\prime} \circ p=1_{A / S}$, the sequence $0 \rightarrow S \rightarrow A \rightarrow A / S \rightarrow 0$ splits and thus $S$ is a summand of $A$.

Dualising 4.3, we obtain a corresponding statement for quasiinjectives.

Lemma 4.3'. Let $S$ be a submodule of a quasi-injective module $A$. Then $S$ will be a summand if and only if $S$ is isomorphic to a summand of $A$.

REMARK. Lemma 3.2 and $3.2^{\prime}$ can also be easily deduced from 4.3 and $4.3^{\prime}$ respectively.

Lemma 4.4. Let $A$ be a quasi-projective module. Then the exact

2) $g \mid B$ denotes the restriction of the map $g$ to $B$. 
sequence $0 \rightarrow T \rightarrow S \stackrel{f}{\rightarrow} A \rightarrow 0$ splits, whenever $S$ is a submodule of $A$.

Proof. Let $g: A \rightarrow A / T$ be an epimorphism such that $g \mid S=f$. Let $h: A \rightarrow A / T$ be monic with $\operatorname{Im} h=S / T$. Then there exists an endomorphism $h^{\prime}$ of $A$ satisfying $h^{\prime} \circ g=h$. Since $\operatorname{Im} h^{\prime}=S$, it is readily seen that $h^{\prime}$ is a split map of the sequence $0 \rightarrow T \rightarrow S \stackrel{f}{\rightarrow} A \rightarrow 0$. Hence the Lemma.

Dualising 3.4, we obtain an analogous property of quasi-injectives.

Lemma 4.4'. If $A$ is quasi-injective, then the exact sequence $0 \rightarrow A \stackrel{i}{\rightarrow} X \rightarrow Y \rightarrow 0$ splits whenever $X$ is a quotient of $A$.

As an easy application of Lemma 4.4 we show that big torisonfree quasi-projectives over an integral domain are projective.

THEOREM 4.5. Let $R$ be a ring without zero divisors. Then any torsion-free quasi-projective $R$-module containing an $R$-independent subset of cardinality exceeding the cardinality of $R$ is projective.

We may assume, without loss in generality, that $R$ is infinite (since otherwise $R$ becomes a field). Let $A$ be a quasi-projective torsion-free $R$-module and $S$ a maximal $R$-independent subset with $|S| \geqq|R|$. Let $F$ be the (free) submodule generated by $S$. Then $|A|=|S| \cdot|R|=|S|$ and so $A$ can be obtained as an epimorphic image of $F$. Since $F$ is free, $A$ is projective by Lemma 4.4 .

REMARK. (i) From the proof of 4.5 it is clear that, if $R$ has no zero divisors, then a torsion-free quasi-projective $R$-module $A$ is projective exactly when $\bigoplus_{m} A$ is quasi-projective for every cardinal $m$.

(ii) K. H. Fuller and D. A. Hill (Notices, Amer. Math. Soc., 16 (1969) 961) show that if $A$ is finitely generated quasi-projective, then $\bigoplus_{m} A$ is quasi-projective for any $m$. An immediate deduction from (i) above: If $R$ has no zero divisors, then a finitely generated torsionfree quasi-projective $R$-module is projective.

COROLlary 4.6. A quasi-projective module over a ring without zero divisors is projective if and only if it is torsion-free and possesses a projective cover.

We need only to prove the "if" part. Let $A$ be torsion-free quasi-projective and $A \cong P / S, P$ projective and $S$ small. By Lemma 4.2, $S$ is fully invariant in $P$. If $m$ denotes the cardinality of $R$, 
then $\bigoplus_{m} A \cong\left(\bigoplus_{m} P\right) /\left(\bigoplus_{m} S\right)$ is quasi-projective, since $\bigoplus_{m} S$ is fully invariant in $\bigoplus_{m} P$. The projectivity of $A$ then follows from Thorem 4.5 .

REMARK. One can deduce that over a ring without zero divisors a quasi-projective module with a projective cover is either torsion or torsion-free. For, suppose $A, P$ and $S$ are as in the preceeding proof and $A$ contains a torsion-free element $a \neq 0$. If $m>\boldsymbol{\aleph}_{0} \cdot|R| \cdot|A|$, then $\bigoplus_{m} A$ is quasi-projective, has cardinality $m$ and contains a free submodule $F$ of rank $m$. By Lemma 4.4, $\bigoplus_{m} A$ and hence $A$ is projective (and torsion-free).

The following theorem characterises Artin Semisimple rings by means of quasi-projectives.

THEOREM 4.7. The following properties are equivalent for any $\operatorname{ring} R$ :

(i) $R$ is Artin Semi-simple.

(ii) The $R$-modules with a projective cover are precisely the quasi-projectives.

(iii) Every quasi-projective $R$-module is projective.

Proof. Trivially (i) implies (ii).

Assume (ii). Let $Q$ be quasi-projective. By assumption $Q$ possesses a projective cover $P$. Then $P \oplus Q$ will have a projective cover and hence is quasi-projective by hypothesis. Lemma 3.2 then implies that $Q$ is projective.

Assume (iii). Since any simple $R$-module is quasi-projective, it becomes projective by assumption. Then all the maximal left ideals of $R$ are direct summands of the left $R$-module $R$ and since $R$ has 1 , we conclude that $R$ is Artinian Semi-simple. This completes the proof.

REMARK 1. Observe that if every $R$-module is quasi-projective then, by Lemma 3.2, $R$ satisfies the condition (iii) above and hence $R$ is Artinian Semi-simple.

REMARK 2. Johnson and Wong [9] showed that the quasi-injective modules over any ring $R$ are exactly the fully invariant submodules of injective $R$-modules. A natural question is whether this can be dualised to quasi-projectives. Precisely, must every quasi-projective $R$-module $A$ be of the form $P / S$ with $P$ projective and $S$ fully invariant in $P$ ? Jans and $\mathrm{Wu}[14]$ answered this in the affirmative under the assumption that $A$ has a projective cover. In the general case, 
the answer turns out to be in the negative. To see this, consider $M=\oplus(Z / p Z)$, where $Z$ is the ring of integers, $\oplus$ is a $Z$-module direct sum and $p$ runs over the set of all primes in $Z$. Clearly $M$ is a quasi-projective $Z$-module [7]. But $M$ cannot be written as $P / S$, where $P$ is a projective (hence free) abelian group and $S$ fully invariant in $P$, since the only fully invariant subgroups of a free abelian group $F$ are of the form $n F, n=1,2, \cdots$.

REMARK 3. In the statement of the Theorem 4.7 (ii), if we replace "precisely" by "necessarily", we obtain a characterisation of Jacobson semi-simple rings: $A$ ring $R$ is Jacobson semi-simple if and only if the $R$-modules possessing projective covers are necessarily quasi-projective. To see this, assume the "if" part. Then, by Lemma 4.2, the small submodules of any projective $R$-module $P$ are fully invariant in $P$. In particular, let $P=R_{1} \oplus R_{2}$ with $R_{i}=R$ and let $J_{i}=J$, the Jacobson radical of $R$, for $i=1,2$. Now $J_{1}$ is small in $R_{1}$ and hence in $P$. But then $J_{1}$ would be fully invariant in $P$, an impossibility since $J_{1}$ can be mapped onto $J_{2}$ by an endomorphism of $P$. Thus $J_{1}=0$ and $R$ is Jacobson Semi-simple. The converse follows on noting that if $R$ is Jacobson Semi-simple, then 0 is the only small submodule of any projective $R$-module.

5. Quasi-projectives over Dedekind domains. In this section we propose to describe the quasi-projective modules over an arbitrary Dedekind domain $R$. First, observe that if $A$ is any quasi-projective $R$-module, then any exact sequence $0 \longrightarrow S \stackrel{i}{\rightarrow} A \stackrel{j}{\rightarrow} A / S \rightarrow 0$ yields the following two exact sequences.

$$
\begin{aligned}
& 0 \longrightarrow \operatorname{Hom}_{R}(A, S) \stackrel{i^{\prime}}{\longrightarrow} \operatorname{Hom}_{R}(A, A) \stackrel{j^{\prime}}{\longrightarrow} \operatorname{Hom}_{R}(A, A / S) \longrightarrow 0 \\
& 0 \longrightarrow \operatorname{Ext}_{R}^{1}(A, S) \stackrel{i^{\prime \prime}}{\longrightarrow} \operatorname{Ext}_{R}^{1}(A, A) \stackrel{j^{\prime \prime}}{\longrightarrow} \operatorname{Ext}_{R}(A, A / S) \longrightarrow 0 .
\end{aligned}
$$

We first consider the torsion free quasi-projective modules. To avoid the trivial situations, the integral domains that we consider are not fields, unless explicitly stated.

LemMa 5.1. Let $R$ be a Dedekind domain. Then the quotient field $K$ of $R$ is a quasi-projective $R$-module if and only if $R$ is a complete discrete valuation ring.

Proof. Suppose $K$ is quasi-projective. Given any $f \in \operatorname{Hom}_{R}(K / R$, $K / R)$, there exists a $f^{\prime} \in \operatorname{Hom}_{R}(K, K)$ such that $f^{\prime} \circ j=j \circ f$ where $j$ is the natural map from $K$ onto $K / R$. Let $f^{\prime \prime}=f^{\prime} \mid R$. Since $R f^{\prime} \subseteq$ $R, f^{\prime \prime}$ is given by a multiplication by an element of $R$. It is readily 
seen that the association $f \mapsto f^{\prime \prime}$ gives an isomorphism of $\operatorname{Hom}_{R}(K / R$, $K / R$ ) onto $R$. Now the exact sequence $0 \rightarrow R \rightarrow K \rightarrow K / R \rightarrow 0$ yields an exact sequence

$$
\begin{aligned}
& \operatorname{Hom}_{R}(K / R, K)=0 \longrightarrow \operatorname{Hom}_{R}(K / R, K / R) \longrightarrow \operatorname{Ext}_{R}^{1}(K / R, R) \\
& \longrightarrow \operatorname{Ext}_{R}^{1}(K / R, K)=0
\end{aligned}
$$

(the first term is zero since $K / R$ is torsion and $K$ is torsion-free). Thus $R \cong \operatorname{Hom}_{R}(K / R, K / R) \cong \operatorname{Ext}_{R}^{1}(K / R, R)$ and the Corollary 7.9 of [13] implies that $R$ is a complete discrete valuation ring.

Conversely, suppose $R$ is a complete discrete valuation ring. Then any $R$-submodule $S$ of $K$ is isomorphic to $R$ or $K$ and hence, by Theorem 7.9 of [13], $\operatorname{Ext}_{R}^{1}(K, S)=0 . K$ is then clearly quasiprojective.

We shall first describe the torsion-free quasi-projectives over Dedekind domains which are not complete discrete valuation rings.

Lemma 5.2. Suppose $R$ is a Dedekind domain which is not a complete discrete valuation ring. Then any torsion-free quasiprojective $R$-module $A$ is torsionless.

Proof. Let $0 \neq x \in A$ and $S$ the pure submodule generated by $x$. Since $R$ is not a complete discrete valuation ring, $A$ (and therefore $S$ ) is reduced, by Lemma 3.1. Thus $S \neq P S$ for some prime ideal $P$ of $R$. Then $S / P S$, being bounded and pure, is a summand of $A / P S$ (Theorem 5 [11]). A nonzero cyclic summand of $S / P S$ will be isomorphic to $R / P$ and can be written as $R y / P y$, for some $y \in S$. Let $g: S / P S \rightarrow R y / P y$ be a nonzero map. Consider the following diagram

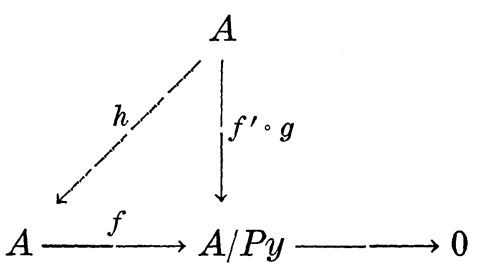

where $f^{\prime}: A \rightarrow S / P S$ is obtained via the projection $A / P S \rightarrow S / P S$ and $f$ is the natural map. By the quasi-projectivity of $A$, there exists $h: A \rightarrow A$ making the diagram commutative. Now $A(h \circ f)=A\left(f^{\prime} \circ g\right) \subseteq$ $R y / P y$, so that $A h \cong R y$. Thus $h: A \rightarrow R y \cong R$ and $x h \neq 0$ since $h$ does not vanish on the rank 1 submodule $S$. It follows that $A$ is torsionless. 
CoRollary 5.3. Let $R$ be a Dedekind domain which is not a complete discrete valuation ring. Then any torsion-free $R$-module $A$ is $\aleph_{1}$-projective. Hence any torsion-free $R$-module of atmost countable rank is projective.

Proof. Let $S$ be a submodule of $A$ of rank 1. By Lemma 5.2, $A$ is torsionless so that for each $a \neq 0$ in $S$, there exists $f: A \rightarrow R$ such that $a f \neq 0$. Since $S$ has rank 1 and im $f$ is torsion-free, $f \mid S$ is mono. As $R$ is hereditary, $S$ is projective. By finite induction, it is clear that any submodule of $A$ of finite rank is projective. Then a well-known step-wise argument (see for example Lemma 8.3.1 [13]) yields that any submodule of countable rank of $A$ is projective.

In the following $\sigma$ denotes cardinality of the set of all distinct prime ideals of $R$.

Proposition 5.4. Let $R$ be a Dedekind domain. Then any torsion-free quasi-projective of rank $m \geqq \sigma \aleph_{0}$ is projective.

Proof. Let $A$ be a torsion-free $R$-module of $\operatorname{rank} m \geqq \sigma \aleph_{0}$ and $K$ be the quotient field of $R$. It is easy to see that $R\left(P^{\infty}\right)$ is countably generated. Now $K / R$ is $\bigoplus_{P} R\left(P^{\infty}\right)$, where $P$ runs over the set of distinct non-zero prime ideals of $R$ and hence $K$ has a generating set of cardinality $\sigma \boldsymbol{\aleph}_{0}$. If $D$ is an injective hull of $A$, then $D \cong \bigoplus_{m} K$ has a generating set of cardinality $m$. It is then readily seen that $A$ itself is generated by $m$ elements. Let $F$ be a free submodule of $A$ of rank $m$ (for example $F$ may be the submodule generated by a maximal $R$-independent subset of $A$ ). $A$ can be got as an epimorphic image of $F$ and hence by Lemma 4.4, $A$ is a direct summand of $F$ and hence projective.

Combining 5.3 and 5.4, we get the following.

Theorem 5.5. Let $R$ be a Dedekind domain which is not a complete discrete valuation ring and $\sigma \leqq \boldsymbol{\aleph}_{0}$. Then a torsion-free $R$ module is quasi-projective if and only if it is projective.

REMARK. If we assume the continuum hypothesis and use 5.3 and 5.4, then we can sharpen 5.5 to the following: Let $R$ be $a$ Dedekind domain wich is not a complete discrete valuation ring and $\sigma \leqq 2^{\aleph_{0}}$. Then any torsion-free quasi-projective $R$-module is projective.

Next we consider the case when $\sigma>2^{\aleph_{0}}$.

Proposition 5.6. Let $R$ be a Dedekind domain and $A$ be $a$ 
torsion-free quasi-projective $R$-module of infinite rank $m$. Then $A$ contains a free summand of rank $m$.

Proof. Let $P$ be any non-zero prime ideal of $R . R\left(P^{\infty}\right)$ is a countably generated injective $R$-module. If $Q=\bigoplus_{m} R\left(P^{\infty}\right)$, then, as $R$ is Noetherian, $Q$ is an injective $R$-module. Clearly $Q$ has a generating set. of cardinality $m$. Let $F$ be the free-submodule generated by a maximal $R$-independent subset of $A$. Then $Q$ can be obtained as a quotient of $F, Q \cong F / S$ for some submodule $S$. Consider the following diagram,

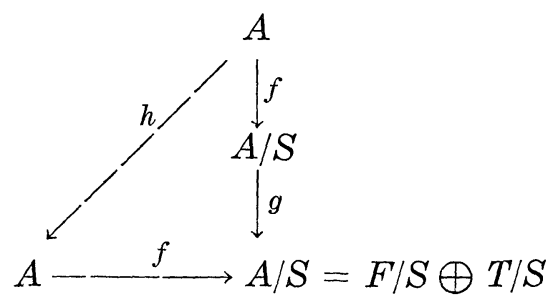

where $g: A / S \rightarrow F / S$ is a projection of $A / S$ onto the injective summand $F / S$ and $f$ is the natural map. By the quasi-projectivity of $A$, there exists $h: A \rightarrow A$ such that $h \circ f=f \circ g$. It is clear that $A h \leqq F$ and since $R$ is hereditary $A h$ is projective. As $F / S$ is a direct sum of $m$ copies of $R\left(P^{\infty}\right)$, it is clear that the rank of $A h=m$. Thus $A=$ $F^{\prime} \oplus K$, where $K$ is the kernel of $h$ and $F^{\prime}$ is a projective module of infinite rank $m$ and hence is free [11].

Combining 5.3, 5.4 and 5.6 we get,

THeOREM 5.7. Let $R$ be a Dedekind domain with $\sigma>2^{\aleph_{0}}$. Then any torsion-free quasi-projective $R$-module $A$ is projective if either (i) $\operatorname{rank} A \leqq \boldsymbol{\aleph}_{0}$ or (ii) $\operatorname{rank} A \geqq \sigma$. In the case when $\boldsymbol{\aleph}_{0}<\operatorname{rank} A<\sigma$, $A$ is torsionless, $\boldsymbol{\aleph}_{1}$-projective and contains a free summand $F$ having the same rank as $A$.

The following theorem characterises torsion-free quasi-projectives over a complete discrete valuation ring.

THEOREM 5.8. Suppose $R$ is a complete discrete valuation ring. Then the torsion-free quasi-projective $R$-modules are just the free $R$ modules and the torsion-free $R$-modules of finite rank.

Proof. By Kaplansky [10], any torsion-free $R$-module of finite rank is of the form $\left(\bigoplus_{i=1}^{n} K_{i}\right) \oplus\left(\bigoplus_{j=1}^{m} R_{j}\right)$ where each $R_{j} \cong R$ and each $K_{i} \cong K$, the quotient field of $R$. Thus if $A$ is any finite rank torsion-free $R$-module and $S$ is any submodule, then both are direct 
sums of finite number of copies of $K$ and $R$, so that

$$
\operatorname{Ext}_{R}^{1}(A, S) \cong \bigoplus_{r} \operatorname{Ext}_{R}^{1}(K, R),
$$

where $r$ is finite. By Lemma 5.1, $K$ is quasi-projective so that

$$
\operatorname{Ext}_{R}^{1}(K, R)=0 \text {. }
$$

Thus $\operatorname{Ext}_{R}^{1}(A, S)=0$, whence $\operatorname{Hom}_{R}(A, A) \stackrel{f^{\prime}}{\rightarrow} \operatorname{Hom}_{R}(A, A / S) \rightarrow 0$ is exact for every submodule $S$ of $A$, where $f^{\prime}$ is induced by the natural map $f: A \rightarrow A / S$. The quasi-projectivity of $A$ then follows. On the other hand if $A$ is a torsion-free quasi-projective $R$-module of infinite rank, then by Proposition 5.4, $A$ is projective and hence free.

Corollary 5.9. If $A$ is quasi-projective, then a direct sum $\oplus A$ of copies of $A$ need not be quasi-projective.

ExAmple. Suppose $A$ is any torsion-free module of finite rank over a complete discrete valuation ring $R$ such that $A$ is not projective (for example $A=K$, the quotient field of $R$ ). Then any finite direct sum of copies $A$ is quasi-projective but, by 5.8, no direct sum of infinite number of copies of $A$ can be quasi-projective.

We shall now describe the torsion quasi-projectives over $R$.

Theorem 5.10. A torsion module $A$ over a Dedekind domain $R$ is quasi-projective if and only if each $P$-primary component $A_{P}$ is a direct sum copies of the same cyclic module $R / P^{k}$ for some fixed positive integer $k$ depending on $P$.

Proof. Since a $P$-primary module over $R$ can be viewed as a module over the principal ideal domain $R_{P}$, and quasi-projectivity survives under this transition, we may assume that $R$ itself is a principal ideal domain. Our proof would be sketchy since it is similar to the one given in [7]. Now $R\left(P^{\infty}\right)$ is not quasi-projective since otherwise, by Lemma 4.3, every submodule of $R\left(P^{\infty}\right)$ would be a summand. Thus a torsion quasi-projective $R$-module $A$ is necessarily reduced. Again, by Lemma 4.3, $A$ cannot contain a summand of the form $\left(R / P^{k_{1}}\right) \oplus\left(R /\left(P^{k_{2}}\right)\right)$ with $k_{1}>k_{2}$, since there is an epimorphism $R /\left(P^{k_{2}}\right) \rightarrow R /\left(P^{k_{1}}\right)$ whose kernel is not a summand. Thus the basic submodules $B_{P}$ (see [6]) of each $P$-primary component $A_{P}$ are bounded and since the $A_{P}$ are reduced, each $A_{P}$ coincides with $B_{P}$ which is clearly a direct sum of isomorphic cyclic modules. The "only if" part follows.

Conversely, if $A$ is a direct sum $\bigoplus_{m} R /\left(P^{k}\right)$ of isomorphic cyclic modules, then $A \cong F / P^{k} F$, where $F$ is free, say, $F=\bigoplus_{m} R$. Since 
$P^{k} F$ is fully invariant in $F, A$ is quasi-projective, by 4.1 .

Corollary 5.11. A torsion module $A$ over a Dedekind domain $R$ is quasi-projective if and only if $A$ is quasi-injective but not injective.

Proof. By Johnson and Wong [9], the quasi-injectives are precisely the fully invariant submodules of injective modules. The corollary then follows on noting that $P$-primary injective $R$-modules are direct sums of copies of $R\left(P^{\infty}\right)$ and their proper fully invariant submodules are direct sums of isomorphic cyclic $P$-primary modules.

The following theorem concludes our investigation of quasi-projectives over Dedekind domains.

Theorem 5.12. A quasi-projective module over a Dedekind domain is either torsion or torsion-free.

Proof. Suppose $A$ is a quasi-projective $R$-module with its maximal torsion submodule $A_{t} \neq 0$. Since $R\left(P^{\infty}\right)$ is not quasi-projective for any prime ideal $P, A_{t}$ is reduced and thus $A$ has torsion cyclic summands [11]. Let $A=\left(R / P^{k}\right) \oplus B$. Now if $R$ is not a complete discrete valuation $\operatorname{ring}, B / B_{t}$ is torsion-free quasi-projective and hence is torsionless $(5.2)$ so that $B$ has a projective summand $I$ of rank 1. If $R$ is a complete discrete valuation ring, then as in the proof of 5.10, one can then show that $B_{t}=B_{P}$ is a bounded direct sum of isomorphic cyclic modules, where $P$ is the unique nonzero prime ideal of $R$. Hence $B=B_{P} \oplus B / B_{P}$, so $B / B_{P}$ is a torsion-free quasi-projective $R$-module and hence contains a summand isomorphic to $R$ or $K$, the quotient field of $R$ (5.8). Thus, in either case, $A$ has a summand of the form $\left(R / P^{k}\right) \oplus C$, where $C \cong K$, the quotient field of $R$ or $C \cong I$, an ideal of $R$. Choose a submodule $S$ of $C$ such that $S \cong R$ or $S \cong I P^{k}$ according as $C \cong K$ or $C \cong I$. Then there exists a nonzero morphism $g: R / P^{k} \rightarrow C / S$. Consider the following diagram.

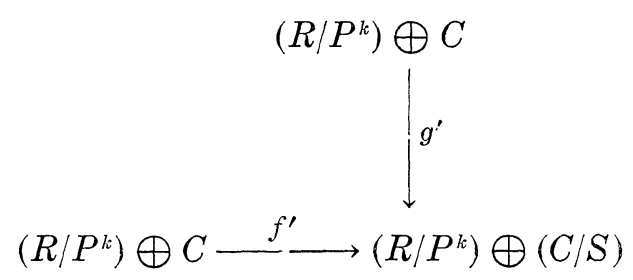

where $f^{\prime}=\left(\begin{array}{ll}1 & 0 \\ 0 & f\end{array}\right), \quad f$ being the natural map and $g^{\prime}=\left(\begin{array}{ll}0 & g \\ 0 & 0\end{array}\right)$, where $g$ is any nonzero homomorphism $R / P^{k} \rightarrow C / S$. This $g^{\prime}$ cannot be lifted to an endomorphism $h$ of $\left(R / P^{k}\right) \oplus C$ satisfying $h \circ f=g^{\prime}$, a 
contradiction. We thus conclude that $A$ is either torsion or torsionfree.

6. Perfect rings. In this section perfect rings are characterised by means of quasi-projective $R$-modules.

THEOREM 6.1. Let $R$ be any ring. Then the following properties are equivalent.

(i) $R$ is left perfect.

(ii) $A$ direct limit of quasi-projective left $R$-modules is quasiprojective.

(iii) A direct limit of finitely generated quasi-projectives over $R$ is quasi-projective.

(iv) Any flat left $R$-module is quasi-projective. ${ }^{3)}$

Proof. Let $Q=\underset{\lim }{\longrightarrow} Q_{i}, i \in I$ where $I$ is a directed set and the $Q_{i}$ 's are quasi-projective $R$-modules. To each $i \in I$, there exists, by hypothesis, an exact sequence $0 \rightarrow K_{i} \stackrel{u_{i}}{\rightarrow} P_{i} \stackrel{v_{i}}{\rightarrow} Q_{i} \rightarrow 0$ where $P_{i}$ is projective and $K_{i}$ is small in $P_{i}$. Now $\left\{P_{i}\right\}_{i \in I}$ and $\left\{K_{i}\right\}_{i \in I}$ can be made into directed systems in a natural way so that we get a directed system of exact sequences. Let $K=\lim K_{i}$ and $P=\lim P_{i}$. Suppose for each $i \in I$

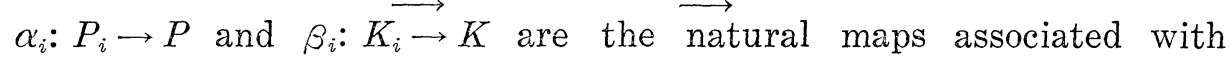
the direct limits. Since the direct limit commutes with exact sequences, $0 \rightarrow K \stackrel{u}{\rightarrow} P \stackrel{v}{\rightarrow} Q \rightarrow 0$ is exact. We have the following commutative diagram:

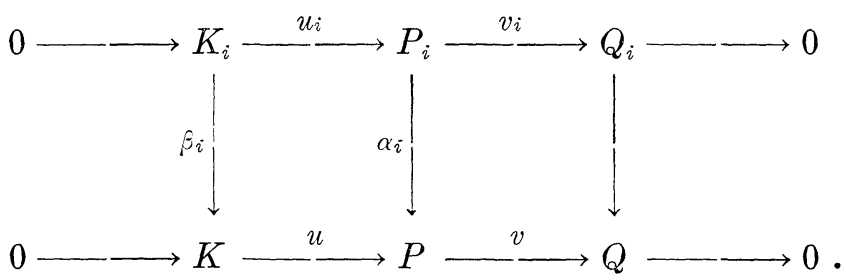

We claim that $K u$ is fully invariant in $P$. Let $f \in \operatorname{End}_{R}(P)$ and $k \in K$. As $R$ is perfect, $P$ is a direct sum of cyclic projective $R$-modules [12]. Let $P^{\prime}$ be a finitely generated summand of $P$ containg $(k) u$ and let $P \stackrel{g}{\rightarrow} P^{\prime}$ be the natural projection. As $\left(P^{\prime}\right) f$ is finitely generated, we can choose a $j \in I$ and a $k_{j} \in K_{j}$ such that $\left(P_{j}\right) \alpha_{j} \supset\left(P^{\prime}\right) f$ and $\left(k_{j}\right) \beta_{j}=k$. Consider the following diagram:

3) In a private communication Dr. J. Golan has indicated that he has also proved the equivalence of (iv) and (i). 


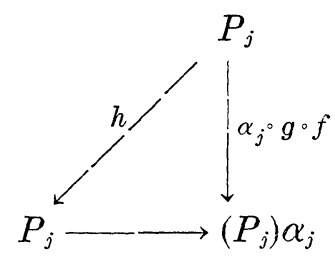

where $h$ exists by the projectivity of $P_{j}$. As $\left(K_{j}\right) u_{j}$ is fully invariant in $P_{j}$ (by 4.2 ), $\left(k_{j}\right) u_{j} h \in\left(K_{j}\right) u_{j}$. Now

$$
\begin{aligned}
(k) u \circ f & =(k) u \circ g \circ f\left(\operatorname{as~} g \mid P^{\prime}=1_{P^{\prime}}\right)=\left(k_{j}\right) \beta_{j} \circ u \circ g \circ f=\left(k_{j}\right) u_{j} \circ \alpha_{j} \circ g \circ f \\
& =\left(k_{j}\right) u_{j} \circ h \circ \alpha_{j} \in\left(K_{j}\right) u_{j} \circ \alpha_{j}=\left(K_{j}\right) \beta_{j} \circ u \subseteq(K) u .
\end{aligned}
$$

Thus $(K) u$ is fully invariant in $P$ whence $Q \cong P /(K) u$ is quasi-projective.

Clearly (ii) $\Rightarrow$ (iii) and, since a flat module is a direct limit of finitely generated projectives, (iii) implies (iv).

Assume (iv). Let $A$ be flat and $P$ projective such that $A \cong P / S$. Since $A \oplus P$ is flat, it is quasi-projective, by hypothesis. Then Lemma 3.2 implies that $A$ is projective. Thus a direct limit of projective left $R$-modules is projective and so $R$ is left perfect, by theorem $P$ of [2]. This proves (i).

REMARK. If $R$ is left perfect and $A$ is a quasi-projective lelf $R$ module, then a direct sum of any number of carbon copies of $A$ is again quasi-projective. This property, however, does not characterize the perfect rings. Indeed, the investigations made in $\S 5$ show that if $R$ is a countable Dedekind domain which is not a complete discrete valuation ring and $A$ is a quasi-projective $R$-module, then $\bigoplus_{m} A$ is quasi-projective for any cardinal number $m$.

7. Generalization. In this section, we consider a weakened form of quasi-projectivity called $w$. quasi-projectives. The $w$.quasiprojective abelian groups were considered in [8]. We give a description of $w$. quasi-projectives over a Dedekind domain. It is also shown that $w$. quasi-perfect abelian categories with enough projectives are perfect.

Definition. An object $A$ in a category $\mathscr{A}$ is called weak quasiprojective (for short, $w$. quasi-projective) if for any epimorphism $f: A \rightarrow B$ and any $g: A / B \rightarrow A / B$, there is a $g^{\prime}: A \rightarrow A$ making the following diagram 


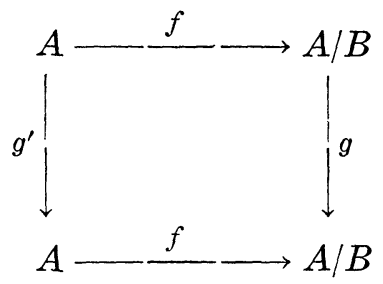

commutative.

It is clear that any quasi-projective is weak quasi-projective. But the converse is not true. The abelian group $Z\left(P^{\infty}\right)$ is $w$. quasiprojective, eventhough it is not a quasi-projective $Z$-module.

We start with the following lemma which gives a criterion for quasi-projectivity. The proof is straight forward and hence is omitted.

Lemma 7.1. An $R$-module $A$ is quasi-projective if and only if $A \oplus A$ is weak quasi-projective.

REMARK. It is clear from 7.1 that, unlike the quasi-projective case, if $A$ is $w$.quasi-projective then $A \oplus A$ need not be $w$.quasiprojective.

The next lemma can be obtained by modifying the arguments of 3.2 .

LEMmA 7.2. [8]. If $A \oplus B$ is w. quasi-projective and there is an epimorphism $f: A \rightarrow B$, then $B$ will be isomorphic to a summand of $A$.

One can define a weak quasi-perfect category in the obvious manner. Using Lemma 7.1 and proceeding exactly as in the proof of Theorem 3.4, we obtain.

THEOREM 7.3. A weak quasi-perfect abelian category with enough projectives is perfect.

If we suitably modify the preceding investigation of the quasiprojectives over a Dedekind domain and make use of Lemma 7.2 we can obtain the following theorem whose proof is omitted.

THEOREM 7.4. Let $R$ be a Dedekind domain.

(i) $A$ torsion $R$-module $A$ is weak quasi-projetive if and only if 
each P-primary component $A_{P}$ is either quasi-projective or $A_{P} \cong R\left(P^{\infty}\right)$.

(ii) If the number $\sigma$ of prime ideals of $R$ is $\leqq 2^{\aleph_{0}}$ then the torsion-free weak quasi-projectives are just the (torsion-free) quasiprojectives. If $\sigma>2^{\mathbf{N}_{1}}$, then a torsionfree weak quasi-projective $R$ module $A$ is projective if either $A$ has rank $\leqq \boldsymbol{\aleph}_{0}$ or (ii) rank $A>\sigma$. If $\boldsymbol{\aleph}_{0}<\operatorname{rank} A<\sigma, A$ is $\boldsymbol{\aleph}_{1}$-projective and contains a free summand $F$ whose rank is equal to rank $A$.

(iii) $A$ properly mixed $R$-module $A$ is weak quasi-projective if and only if $A \cong B \oplus C$ where $B$ is reduced torsion-free quasi-projective of finite rank and $C$ is an injective submodule of $K / R$, where $K$ is the quotient field of $R$.

The authors are indebted to the referee for pointing out a few inaccuracies and for offering many suggestions for improvement.

\section{REFERENCES}

1. B. Banaschewski, Projective Covers in Categories of Topological Spaces and Topological Algebras, McMaster University, 1968.

2. H. Bass, Finitistic dimension and a homological generalisation of semiprimary rings, Trans. Amer. Math. Soc., 95 (1960), 466-488.

3. S. Eilenberg, Homological dimension and Syzygies, Ann. of Maths., 64 (1956), 328336.

4. C. Faith, Review 3817, Math. Reviews, Vol. 36 (1968).

5. P. Freyd, Abelian Categories, Harper and Row, New York, (1964).

6. L. Fuchs, Abelian Groups, Pergamon Press, New York, 1960.

7. L. Fuchs and K. M. Rangaswamy, Quasi-projective abelian groups, Bull. Soc. Math. France, 98 (1970), 5-8.

8. S. Janakiraman, Ph. D. thesis, Madurai University (1970).

9. R. E. Johnson and R. T. Wong, Quasi-injective modules and irreducible rings, J. London Math. Soc., 36 (1961), 260-268.

10. I. Kaplansky, Infinite Abelian Groups, Ann Arbor, (1956).

11. Modules over Dedekind domains and valuation rings, Trans. Amer. Math. Soc., 72 (1952), 327-340.

12. A. Koehler, Ph. D. thesis, Indiana University, (1969).

13. R. J. Nunke, Modules of extensions over a Dedekind ring, Illinois J. Math. 3 (1959), $224-242$.

14. L. E. T. Wu and J. P. Jans, On quasi-projectives, Illinois J. Math. 11 (1967), 439-448.

Received August 1, 1970 and in revised form October 12, 1971. Most of the results in this paper were presented at a seminar held under the chairmanship of Professor N. Jacobson during the Algebra Conference at New Delhi, in April, 1969.

MadURAI UNIVERSity

AND

The Australian National University

Canberra, A.C.T. 2600, Australia

AND

The UNIVERSity of Bombay

Santa Cruz (East), Bombay-28, India 


\section{PACIFIC JOURNAL OF MATHEMATICS}

\section{EDITORS}

\section{H. SAMElson}

Stanford University

Stanford, California 94305

C. R. Новву

University of Washington

Seattle, Washington 98105
J. DugunduI

Department of Mathematics University of Southern California

Los Angeles, California 90007

RICHARD ARENS

University of California

Los Angeles, California 90024

\section{ASSOCIATE EDITORS}
E. F. BECKENBACH
B. H. NeUmanN
F. WOLF
K. YosHIDA

\section{SUPPORTING INSTITUTIONS}

UNIVERSITY OF BRITISH COLUMBIA

UNIVERSITY OF SOUTHERN CALIFORNIA

CALIFORNIA INSTITUTE OF TECHNOLOGY

STANFORD UNIVERSITY

UNIVERSITY OF CALIFORNIA

UNIVERSITY OF TOKYO

MONTANA STATE UNIVERSITY

UNIVERSITY OF UTAH

UNIVERSITY OF NEVADA

WASHINGTON STATE UNIVERSITY

NEW MEXICO STATE UNIVERSITY

OREGON STATE UNIVERSITY

UNIVERSITY OF OREGON

OSAKA UNIVERSITY

UNIVERSITY OF WASHINGTON

* *

AMERICAN MATHEMATICAL SOCIETY

NAVAL WEAPONS CENTER 


\section{Pacific Journal of Mathematics}

\section{Vol. 43, No. $1 \quad$ March, 1972}

Alexander (Smbat) Abian, The use of mitotic ordinals in cardinal

arithmetic ....................................... 1

Helen Elizabeth. Adams, Filtrations and valuations on rings ......... 7

Benno Artmann, Geometric aspects of primary lattices .............. 15

Marilyn Breen, Determining a polytope by Radon partitions ........... 27

David S. Browder, Derived algebras in $L_{1}$ of a compact group .......... 39

Aiden A. Bruen, Unimbeddable nets of small deficiency .............. 51

Michael Howard Clapp and Raymond Frank Dickman, Unicoherent

compactifications ............................... 55

Heron S. Collins and Robert A. Fontenot, Approximate identities and the strict topology ................................... 63

R. J. Gazik, Convergence in spaces of subsets................. 81

Joan Geramita, Automorphisms on cylindrical semigroups ........... 93

Kenneth R. Goodearl, Distributing tensor product over direct product ..... 107

Julien O. Hennefeld, The non-conjugacy of certain algebras of

operators ................................... 111

C. Ward Henson, The nonstandard hulls of a uniform space ........... 115

M. Jeanette Huebener, Complementation in the lattice of regular

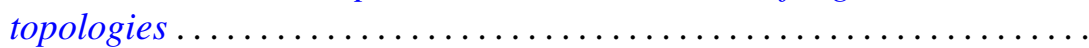

Dennis Lee Johnson, The diophantine problem $Y^{2}-X^{3}=A$ in a

polynomial ring .................................... 151

Albert Joseph Karam, Strong Lie ideals . . . . . . . . . . . . . . . . . . . . 157

Soon-Kyu Kim, On low dimensional minimal sets ............... 171

Thomas Latimer Kriete, III and Marvin Rosenblum, A Phragmén-Lindelöf

theorem with applications to $M(u, v)$ functions ..... . .

William A. Lampe, Notes on related structures of a universal algebra . . . . 189

Theodore Windle Palmer, The reducing ideal is a radical .

207

Kulumani M. Rangaswamy and N. Vanaja, Quasi projectives in abelian and module categories ................................ 221

Ghulam M. Shah, On the univalence of some analytic functions ......... 239

Joseph Earl Valentine and Stanley G. Wayment, Criteria for Banach

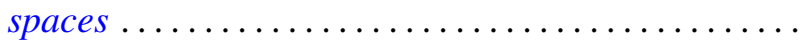

Jerry Eugene Vaughan, Linearly stratifiable spaces ............... 253

Zbigniew Zielezny, On spaces of distributions strongly regular with respect to partial differential operators ..................... 